Supporting Information For

Benzothienobenzothiophene-Based Molecular Conductors: High Conductivity, Large Thermoelectric Power Factor, and One-Dimensional Instability

Yasuhiro Kiyota, ${ }^{\dagger}$ Tomofumi Kadoya, ${ }^{*} \dagger$ Kaoru Yamamoto, ${ }^{*}$ " Kodai Iijima, ${ }^{\dagger}$

Toshiki Higashino, ${ }^{\dagger}$ Tadashi Kawamoto, ${ }^{\dagger}$ Kazuo Takimiya, ${ }^{\ddagger}$ and Takehiko Mori, ${ }^{*}, \dagger, \S$

$\dagger$ Department of Organic and Polymeric Materials, Tokyo Institute of Technology, O-okayama 2-12-1, Tokyo 152-8552, Japan

IDepartment of Applied Physics, Okayama University of Science, Ridaicho 1-1, Okayama 700-0005, Japan

${ }^{\sharp}$ Emergent Molecular Function Research Group, RIKEN Center for Emergent Matter Science (CEMS), Wako, Saitama 351-0198, Japan

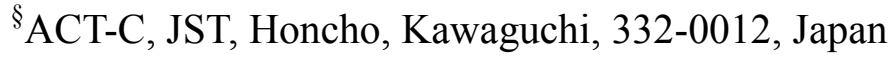




\section{Band Calculation.}

The electronic band dispersions and the Fermi surface are calculated using the intermolecular transfer integrals listed in Table 1 and the tight-binding approximation (Figure S1). ${ }^{30}$

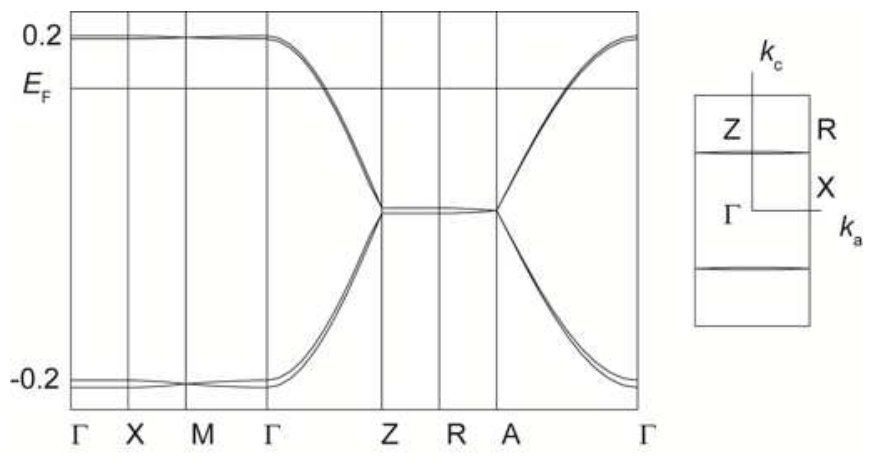

Figure S1. Energy band structure and the Fermi surface of $(\mathrm{BTBT})_{2} \mathrm{AsF}_{6}$.

\section{Conducting properties}

Drift mobility $\mu$ is evaluated from the following equation,

$$
\mu=\frac{\sigma}{n e}
$$

where $\sigma$ is the electrical conductivity, $n$ is the carrier number in a unit cell, and $e$ is the elementary charge.

(BTBT) ${ }_{2} \mathrm{AsF}_{6}$ has the 2:1 composition, so one BTBT molecule has $0.5+$ charge because $\mathrm{AsF}_{6}$ has 1- charge. According to Figure 1, a unit cell includes four BTBT molecules. From these, the drift mobility of the $\mathrm{AsF}_{6}$ salt at room temperature is,

$$
\begin{aligned}
\mu & =\frac{\sigma}{n e} \\
& =\frac{4100 \mathrm{~S} / \mathrm{cm}}{4 \times 0.5 \times \frac{1}{1243 \times 10^{-24} \mathrm{~cm}^{3}} \times\left(1.6 \times 10^{-19} \mathrm{C}\right)} \\
& =15.9 \mathrm{~cm}^{2} / \mathrm{Vs}
\end{aligned}
$$



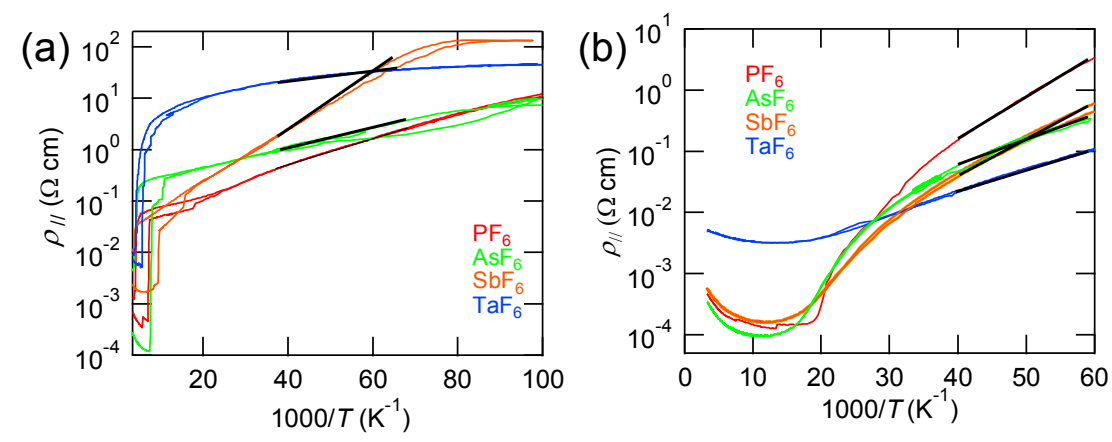

Figure S2. Arrhenius plots of the BTBT salts, (a) the results for free samples and (b) the results for Apiezon coated samples.

Activation energy $E_{\mathrm{a}}$ was calculated from the equation,

$\rho=\rho_{0} \exp \left(\frac{E_{\mathrm{a}}}{k_{\mathrm{B}} T}\right)$

where $\rho$ is the resistivity, $\rho_{0}$ is a constant, $k_{\mathrm{B}}$ is the Boltzmann constant, and $T$ is the temperature. From the straight parts in FigureS2, the $E_{\mathrm{a}}$ were estimated.

The bandwidth is estimated from the Seebeck coefficient according to the one-dimensional tight-binding model.

$S=\frac{\pi^{2} k_{B}^{2} T}{6 e t} \frac{\cos (\pi \rho / 2)}{1-\cos ^{2}(\pi \rho / 2)}$

The Seebeck coefficient is $S=15 \mu \mathrm{V} / \mathrm{K}$ at room temperature, and the charge-transfer degree is $\rho=0.5$.

$4 t=4 \times \frac{(300 \mathrm{~K})}{\left(15 \times 10^{-6} \mathrm{~V} / \mathrm{K}\right)} \times \frac{3.14^{2} \times\left(1.38 \times 10^{-23} \mathrm{~J} / \mathrm{K}\right)^{2}}{6 \times\left(1.6 \times 10^{-19} \mathrm{~J} / \mathrm{V}\right)^{2}} \times 1.414$

This leads to the bandwidth of $4 t=1.38 \mathrm{eV}$. 


\section{ESR measurements}
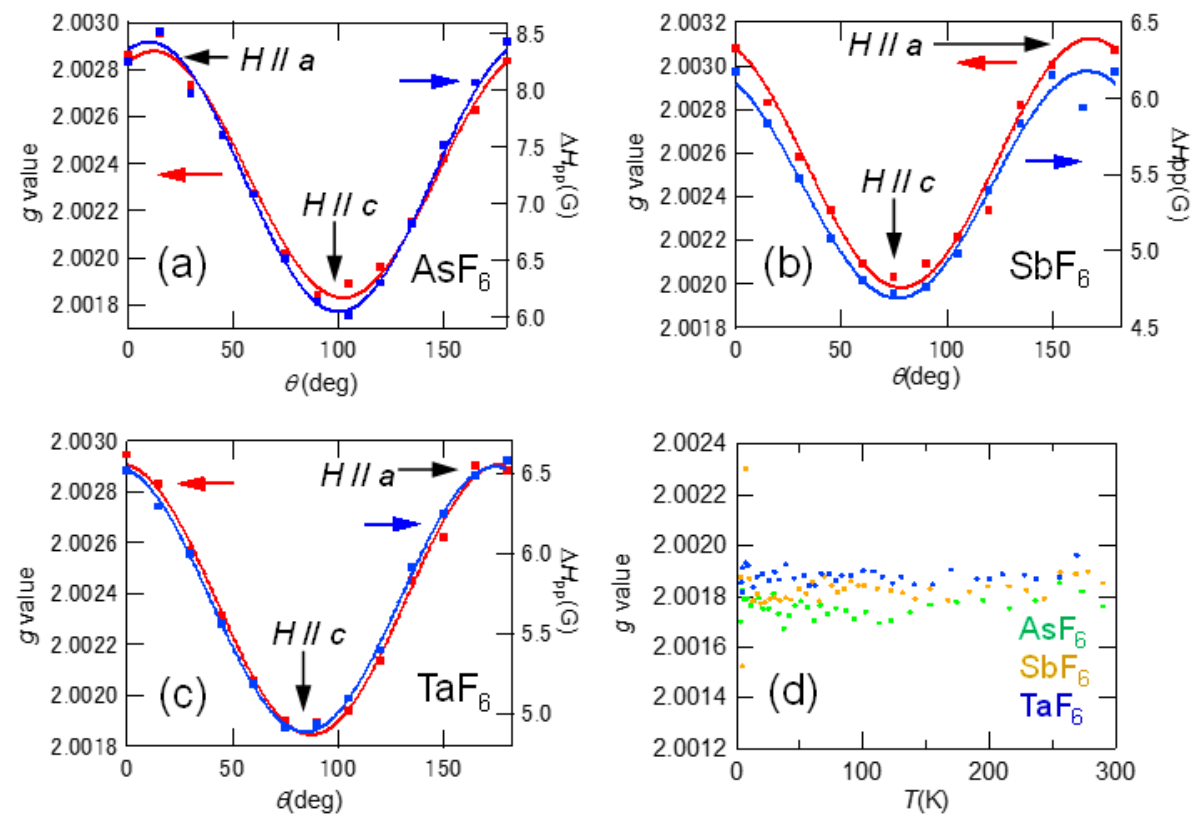

Figure S3. Angle dependence of the $g$ values and the linewidths for the sample rotation around the $a$ axis, for the (a) $\mathrm{AsF}_{6}$, (b) $\mathrm{SbF}_{6}$, and (c) $\mathrm{TaF}_{6}$ salts. (d) Temperature dependence of the $g$ value.

\section{Analysis of the reflectance spectrum of the $\mathrm{PF}_{6}$ salt}

The reflectance spectrum is analysed by assuming the Drude-Lorentz model considering a Drude edge and a single Lorentz oscillator.

$$
\begin{aligned}
& R=\left|\frac{\sqrt{\frac{\tilde{\varepsilon}}{\varepsilon}}-1}{\sqrt{\tilde{\varepsilon}}+1}\right|^{2} \\
& \tilde{\varepsilon}=\varepsilon_{\infty}-\frac{\omega_{p}^{2}}{\omega^{2}+i \omega \Gamma}-\frac{\omega_{p 1}^{2}}{\omega^{2}-\omega_{1}^{2}+i \omega \Gamma_{1}}
\end{aligned}
$$

Here $R$ is the reflectance, $\varepsilon$ is the complex dielectric function, and $\varepsilon_{\infty}$ is the frequency-independent dielectric constant. In addition, $\omega_{\mathrm{P}}$ and $\Gamma$ represent the plasma frequency and the relaxation rate of the charge carriers, respectively, and $\omega_{1}, \Gamma_{1}$, and $\omega_{\mathrm{p} 1}$ 
are the parameters of the Lorentz oscillator. The fitting to the room-temperature spectrum affords $\varepsilon_{\infty}=4.5, \omega_{\mathrm{P}}=11500 \mathrm{~cm}^{-1}, \Gamma=2300 \mathrm{~cm}^{-1}, \omega_{\mathrm{P} 1}=13000 \mathrm{~cm}^{-1}, \omega_{1}=2400 \mathrm{~cm}^{-1}$, and $\Gamma_{1}=2500 \mathrm{~cm}^{-1}$ (Figure S4). From the one-dimensional tight-binding approximation, ${ }^{36}$ the transfer integral is related to $\omega_{\mathrm{P}}$

$$
\omega_{p}^{2}=\frac{4 t a^{2} e^{2} \sin (\pi \rho / 2)}{\pi \varepsilon_{0} \hbar^{2} V_{m}} .
$$

This gives the bandwidth of $4 t_{\mathrm{c}}=1.35 \mathrm{eV}$.

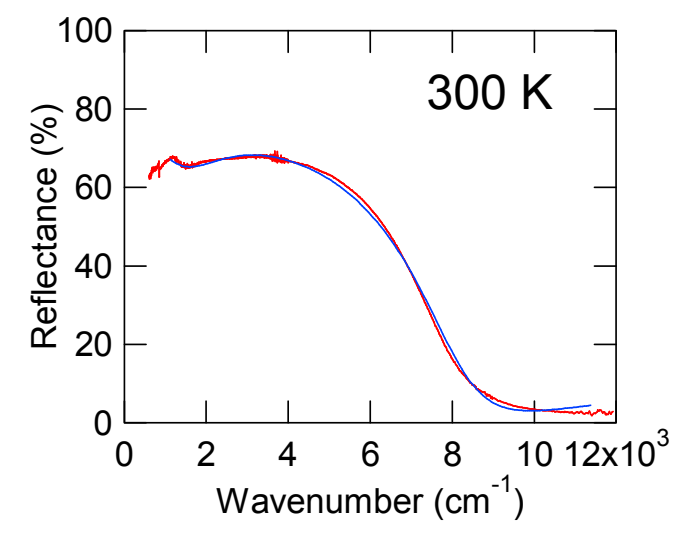

Figure S4. Reflectance spectrum (red) of $(\mathrm{BTBT})_{2} \mathrm{PF}_{6}(E / / c)$ at $300 \mathrm{~K}$ and the fitting curve (blue) based on the Drude and Lorentz model.

\section{DFT calculation of the Raman and IR spectra of the $\mathrm{PF}_{6}$ salt}

For the assignment of the IR and Raman spectra, the density functional calculation is performed for the neutral and monocationic BTBT molecules using Gaussian 09 package at the B3LYP/6-311G(d) level. ${ }^{\text {S1 }}$ The obtained frequencies are adjusted by the scale factor of $0.966{ }^{\text {S2 }}$ The linear electron-molecular vibrational coupling constants are estimated for the neutral state by calculating HOMO energy at a few molecular structures varied along the normal coordinates around the stable geometry. Figure S5 represents the calculated Raman and IR spectra of neutral and monocationic BTBT. Table S1 lists the calculated frequencies of the $a_{g}$ modes appearing in the $\mathrm{C}=\mathrm{C}$ stretching region in comparison with the observed frequencies. The results allow us to assign the Raman peaks as well as the IR e-mv bands (Figure 7a). Since HOMO spreads over the molecule, the Raman shifts are relatively small compared to TTF-type molecules. The $v_{9}$ mode is most suitable to investigate the BTBT charge due to the large Raman shift $(\Delta \omega=47.2)$. 
Table S1. Calculated frequencies $\omega$ of molecular vibrations for the neutral and monocationic BTBT molecules and the e-mv coupling constants $g$ estimated for neutral BTBT. Experimental Raman and IR frequencies are listed together with the fitting parameters used for reproducing the IR spectrum (frequency $\omega$, e-mv coupling constant $g$, and linewidth $\Delta$ ) in Figure $7 \mathrm{~b}$.

\begin{tabular}{|c|c|c|c|c|c|c|c|c|c|}
\hline \multicolumn{5}{|c|}{ DFT Calculation [B3LYP/6-311G(d)] } & \multicolumn{3}{|c|}{ Experiment for $(\mathrm{BTBT})_{2} \mathrm{PF}_{2}$} & \multicolumn{2}{|c|}{ Calcd. IR } \\
\hline \multirow[b]{2}{*}{ Mode } & \multicolumn{3}{|c|}{$\omega\left(\mathrm{cm}^{-1}\right)$} & \multirow{2}{*}{$\begin{array}{l}g\left(\mathrm{~cm}^{-1}\right) \\
\text { Neutral }\end{array}$} & \multicolumn{2}{|c|}{$\omega\left(\mathrm{cm}^{-1}\right)$} & \multirow{2}{*}{$\begin{array}{l}\omega \\
\left(\mathrm{cm}^{-1}\right)\end{array}$} & \multirow{2}{*}{$\begin{array}{l}g \\
\left(\mathrm{~cm}^{-1}\right)\end{array}$} & \multirow{2}{*}{$\begin{array}{l}\Delta \\
\left(\mathrm{cm}^{-1}\right)\end{array}$} \\
\hline & Neutral & Cation & $\Delta \omega$ & & Raman $(100 \mathrm{~K})$ & $\operatorname{IR}(4 \mathrm{~K})$ & & & \\
\hline$v_{5}$ & 1578.0 & 1541.8 & 36.2 & 60 & 1582 & 1568 & 1582 & 100 & 5 \\
\hline$v_{6}$ & 1543.4 & 1539.4 & 4.0 & 145 & 1550 & 1541 & 1550 & 50 & 5 \\
\hline$v_{7}$ & 1473.3 & 1447.9 & 25.4 & 186 & 1479 & 1466 & 1479 & 130 & 10 \\
\hline$v_{8}$ & 1441.8 & 1414.5 & 27.3 & 119 & 1437 & 1431 & 1437 & 70 & 10 \\
\hline$v_{9}$ & 1395.5 & 1348.3 & 47.2 & 68 & 1394 & 1375 & 1394 & 130 & 10 \\
\hline$v_{10}$ & 1301.4 & 1299.2 & 2.2 & 138 & 1325 & 1324 & 1325 & 40 & 10 \\
\hline$v_{11}$ & 1292.0 & 1277.3 & 14.7 & 77 & 1292 & 1281 & 1292 & 150 & 20 \\
\hline$v_{12}$ & 1244.7 & 1216.3 & 28.4 & 0 & 1260 & $1248^{\mathrm{a}}$ & 1260 & 30 & 5 \\
\hline
\end{tabular}

${ }^{\mathrm{a}}$ Antiresonance peak. 


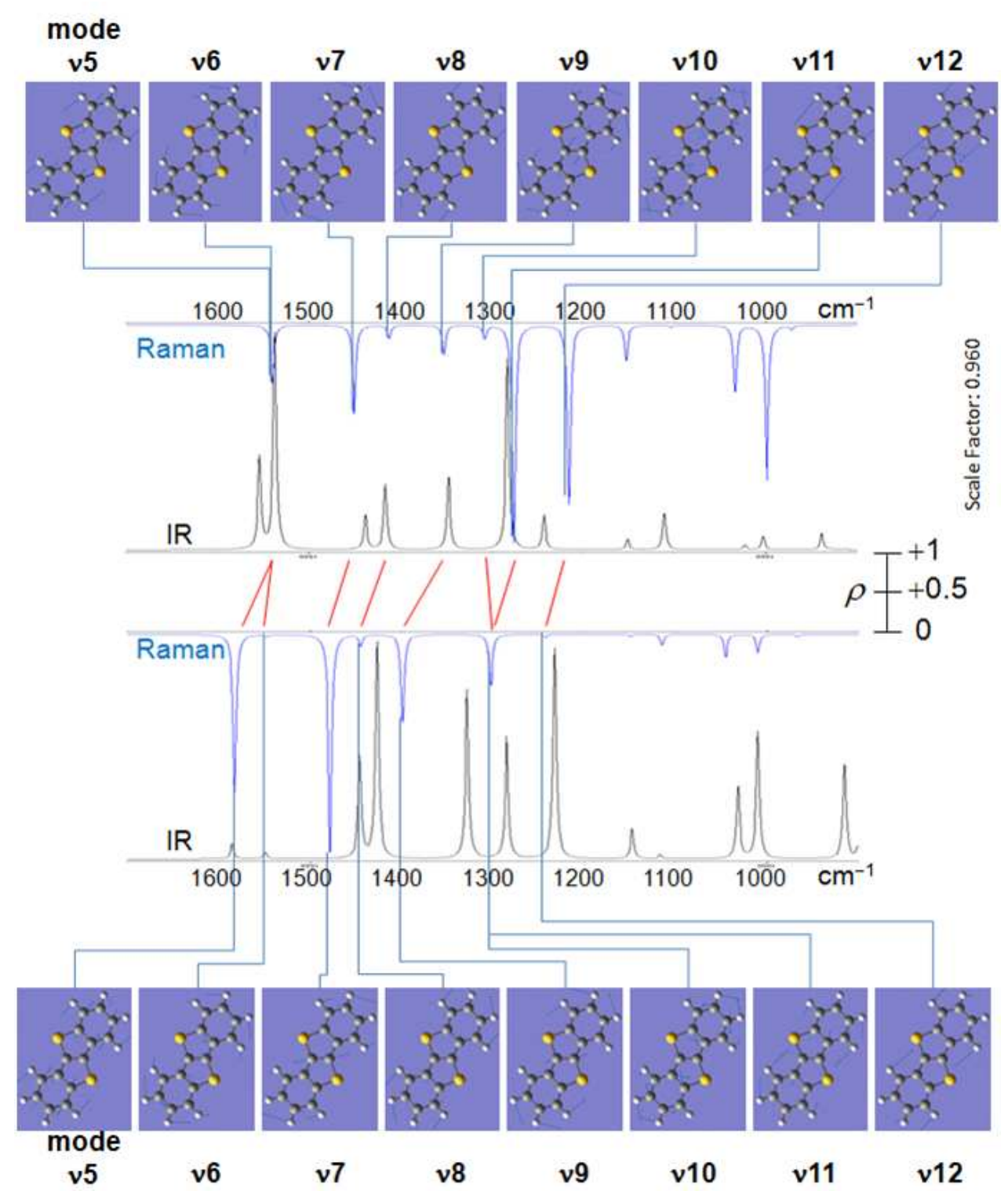

Figure S5. Raman shifts and IR frequency in neutral BTBT and cationic BTBT (1+) calculated using Gaussian 09.

\section{Fitting to the IR spectrum}

A numerical fit to the IR spectrum is carried out using a symmetric dimer model based on the e-mv coupling theory. ${ }^{35}$ The model deals with the antisymmetric mode of molecular $a_{\mathrm{g}}$ vibration as a pseudo electric field that induces charge transfer between the modulated HOMO levels due to the e-mv coupling. The essence of the formulation is 
written by

$$
\sigma(\omega)=-\frac{i \omega C \chi(\omega)}{1-(\chi(\omega) / \chi(0)) D(\omega)}
$$

where $C$ denotes the magnitude of the conductivity, and $\quad \chi(\omega)$ and $D(\omega)$ are defined as

$$
\begin{aligned}
& \chi(\omega)=\frac{\Omega \omega_{\mathrm{CT}}}{\omega_{\mathrm{CT}}^{2}-\omega^{2}-i \omega \Gamma} \text { and } \\
& D(\omega)=\sum\left(\frac{\Omega g_{n}^{2}}{\hbar \omega_{\mathrm{CT}}}\right) \frac{\omega_{n}}{\omega_{\mathrm{n}}^{2}-\omega^{2}-i \omega \gamma_{n}} .
\end{aligned}
$$

Here $\Omega, \omega_{\mathrm{CT}}$, and $\Gamma$ are respectively the matrix element between the ground and excited states, the frequency of the optical gap, and the natural linewidth of the CT band. The vibrational parameters $\omega_{n}$ and $\gamma_{n}$ represent the bare frequency of the $n$-th mode and its linewidth, and $g_{n}$ is the e-mv coupling constant

$$
g_{n}=\sqrt{2 \omega_{n}}\left(\frac{\partial E_{\text {HOMO }}}{\partial Q_{n}}\right)
$$

where $Q_{n}$ denotes the normal coordinate of the $n$-th mode. From the observed CT band, $\omega_{\mathrm{CT}}, \Omega$, and $\Gamma$ are determined. and $\omega_{n}$ are extracted from the corresponding Raman peaks. Then, starting from the calculated values, $g_{n}$ and $\gamma_{n}$ are adjusted so as to reproduce the observed vibronic oscillations. As shown in the broken curve in Figure $7 \mathrm{~b}$, the asymmetric shape and the observed frequencies are satisfactorily reproduced using the parameters in Table S1. The agreement confirms the validity of the density functional calculations and the interpretation of the IR and Raman spectra based on the dimer model.

\section{Symmetry requirements from the IR and Raman spectra}

In the x-ray crystal structure, a unit cell contains four BTBT molecules located on two-fold axes. Then, each molecular vibration splits to four different factor groups. The Raman spectrum is measured using the $a$-polarized excitation and the scattered light is collected by the back scattering $\left(E_{\mathrm{ex}} \perp c\right)$ without an analyzing polarizer. Under this condition, the $A_{1}$ and $B_{1}$ modes are Raman active for the tetragonal symmetry. Thus, the observed doublets, which are observed from room temperature, are understandable as the appearance of these two modes. 
Although this interpretation allows us to explain the doublets qualitatively, the peak separation appears to be larger than the ordinary factor group splitting, thus the large separation should be attributed to the effect of either the e-mv coupling or the uneven charge distribution. In the present case, we believe that the e-mv coupling effect is responsible for the peak separation based on the following discussion.

In the e-mv coupling, the oscillation phase in the adjacent molecules is crucial. In the present crystal symmetry, the $\mathrm{B}_{1}$ mode forms an anti-phase mode in the stacking; the adjacent BTBT molecules oscillate with an opposite phase, leading to a large charge transfer via the coupling effect. The observed peak separation may be ascribed to the factor group splitting enhanced by the e-mv coupling.

(a) Uniform

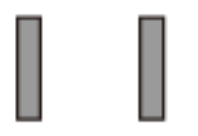

(b) $2 \mathrm{k}_{\mathrm{F}} \mathrm{CDW}$ Tetramer
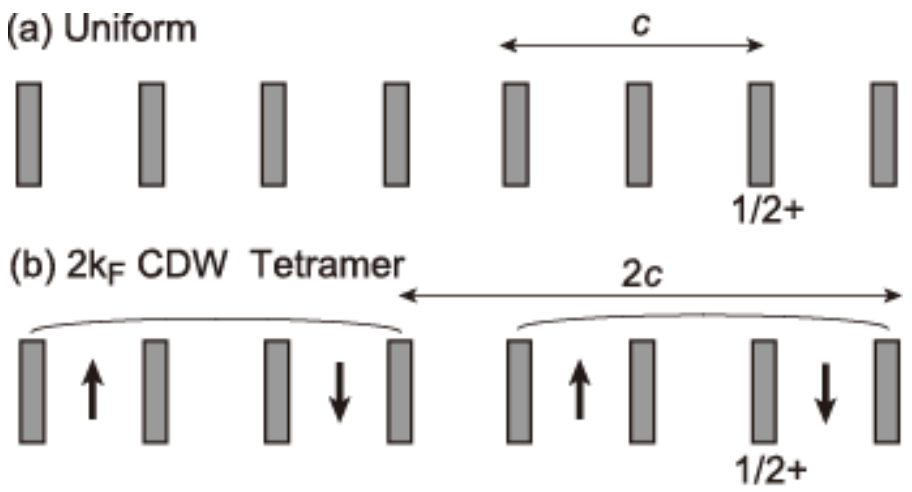

(c) $4 \mathrm{k}_{\mathrm{F}}$ CDW Dimer
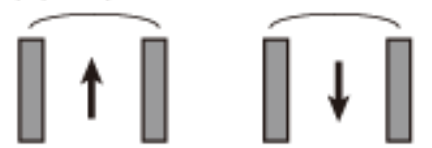

(d) Charge Order
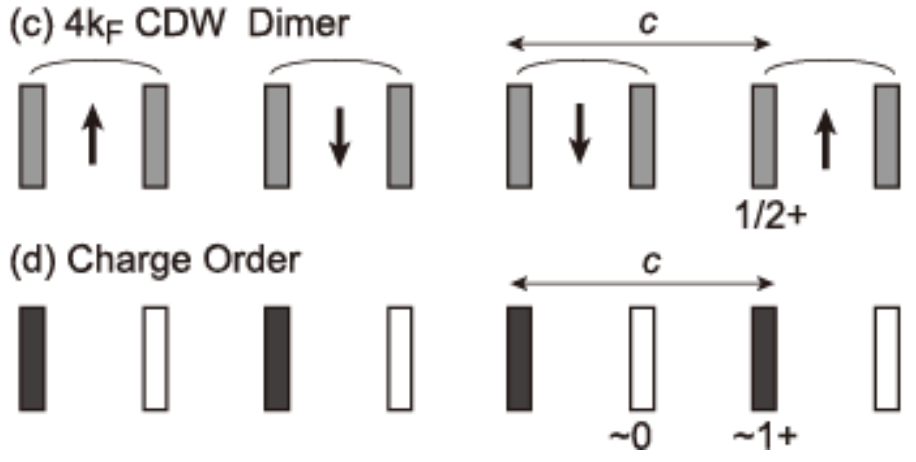

Figure S6. Possible ground states of a BTBT stack.

On the other hand, note that the uniform molecular stacking (Figure S6a) is potentially modulated at low temperatures because the vibronic bands are activated in the IR spectrum. The high- temperature crystal symmetry is incompatible with the molecular displacement, and the actual symmetry is considered to be lower than $P-42{ }_{1} c$. As shown in Figure 7, the 
IR band and the doublet Raman peak appear at different frequencies. This illustrates that the IR peak and the two Raman peaks belong to different normal modes in the lower symmetry. Among the subgroups of $P-42{ }_{1} c$, two symmetries, $P-4$ and $P 2{ }_{1} 2{ }_{1} 2$ are capable of simultaneously satisfying these requirements. Although the latter reduces the crystal symmetry down to orthorhombic, the former retains the tetragonal symmetry. We have analyzed the x-ray diffraction data at $100 \mathrm{~K}$ under the $P-4$ symmetry, and achieved a reasonable convergence as low as that for the $P-42{ }_{1} c$ symmetry. It is therefore suggested that the crystal symmetry is reduced to $P-4$ due to the dimerization in the ground state.

We shall mention the possibility of uneven charge distribution (Figure S6d) as an origin of the peak separation. According to the group theory, there is no subgroup of $P-42{ }_{1} c$ that eliminates the equivalency of the four molecules. Therefore, if the peak separation is a result of the uneven charge distribution, the crystal symmetry is reduced to the monoclinic system $\left(C c, P 2\right.$, or $\left.P 2_{1}\right)$ or the triclinic system $(P 1)$. Although it is difficult to rule out these possibilities experimentally, it should be emphasized that the obtained results, in particular the appearance of the doublets in the Raman spectrum even at room temperature and the activation of the IR band at low temperatures, are explained by the symmetry decrease from the uniform stacking structure with the $P-42{ }_{1} c$ symmetry to the dimerized stacking structure with the $P-4$ symmetry (Figure S6c).

These analyses suggest that the dimeric modulation with even charge distribution develops in the molecular stacking at low temperatures. Taking account of the paramagnetic susceptibility down to low temperatures, the metal-to-insulator transition is presumably attributed to the condensation of $4 k_{\mathrm{F}}$ charge density waves. This is a kind of the Mott insulator phase which emerges owing to the electron Coulomb repulsion, and the instability associated with the spin degree of freedom remains. A slight drop of the susceptibility observed in some cases, ${ }^{\mathrm{S} 3}$ is attributable to the coexistence of $2 k_{\mathrm{F}}$ charge-density waves, which has been theoretically predicted. ${ }^{\mathrm{S} 4}$

\section{REFERENCES}

[S1] Frisch, M. J.; Trucks, G. W.; Schlegel, H. B.; Scuseria, G. E.; Robb, M. A.; Cheeseman, J. R.; Scalmani, G.; Barone, V.; Mennucci, B.; Petersson, G. A.; Nakatsuji, H.; Caricato, M.; Li, X.; Hratchian, H.P.; Izmaylov, A. F.; Bloino, J.; Zheng, G.; Sonnenberg, J. L.; Hada, M.; Ehara, M.; Toyota, K.; Fukuda, R.; Hasegawa, J.; Ishida, M.; Nakajima, T.; Honda, Y.; Kitao, O.; Nakai, H.; Vreven, T.; Montgomery, J. A.; Peralta, Jr., J. E.; 
Ogliaro, F.; Bearpark, M.; Heyd, J. J.; Brothers, E.; Kudin, K. N.; Staroverov, V. N.; Kobayashi, R.; Normand, J.; Raghavachari, K.; Rendell, A.; Burant, J. C.; Iyengar, S. S.; Tomasi, J.; Cossi, M.; Rega, N.; Millam, J. M.; Klene, M.; Knox, J. E.; Cross, J. B.; Bakken, V.; Adamo, C.; Jaramillo, J.; Gomperts, R.; Stratmann, R. E.; Yazyev, O.; Austin, A. J.; Cammi, R.; Pomelli, C.; Ochterski, J. W.; Martin, R. L.; Morokuma, K.; Zakrzewski, V. G.; Voth, G. A.; Salvador, P.; Dannenberg, J. J.; Dapprich, S.; Daniels, A. D.; Farkas, O.; Foresman, J. B.; Ortiz, J. V.; Cioslowski, J.; Fox, D. J. Gaussian 09 (Revision B. 01), Gaussian, Inc., Wallingford, 2009.

[S2] Exploring Chemistry with Electronic Structure Methods, 2nd edition; Foresman, J. B.; Frisch, A. Gaussian Inc.: Pittsburgh 1996.

[S3] Higashino, T.; Kadoya, T.; Kumeta, S.; Kurata, K.; Kawamoto, T.; Mori, T. Eur. J. Inorg. Chem. 2014, 24, 3895.

[S4] Huizinga, S.; Kommandeur, J.; Jonkman, H. T.; Haas, C. Phys. Rev. B, 1982, 25, 1717. 